Ann. Sci. forest., 1968, 25 (3), 135-156.

\title{
INTERGEPTION DES PRÉCIPITATIONS PAR LE COUVERT FORESTIER
}

\author{
G. AUSSENAC \\ avec la collaboration technique de J.-M. Desjeunes \\ Station de Sylviculture et de Production, \\ Centre national de Recherches forestières, 54-Nancy \\ Institut national de la Recherche agronomique.
}

\section{SOMMAIRE}

Les phénomènes d'interception ont été étudiés dans trois peuplements résineux et un peuplement feuillu. Les précipitations arrivant directement au sol sont exprimées en fonction des précipitations incidentes et de l'éclairement relatif. A la suite de ces résultats, une méthode d'évaluation des précipitations sous le couvert est présentée. Enfin, le problème de la signification écologique de l'interception est posé.

\section{1. - INTRODUCTION}

Pendant une période donnée, les gains et les pertes en eau d'un peuplement forestier peuvent être exprimés par l'équation :

$$
P=I+E T+D \pm \Delta S
$$

dans laquelle $P$ : pluviométrie au-dessus des peuplements, $I$ : fraction interceptée des précipitations, $E T$ : évapotranspiration du peuplement considéré, $D:$ perte par drainage et ruissellement superficiel, $\Delta S$ : variation du stock d'eau dans le sol.

L'analyse du devenir des précipitations (rainfall) arrivant sur un peuplement permet de distinguer :

- une fraction qui atteint directement le sol (througfall) ;

- une partie qui s'écoule le long des troncs (stem-flow) et arrive finalement au sol ;

— une fraction qui est arrêtée par le feuillage et les branches. 
C'est l'interception qui peut être évaluée en faisant la différence entre les précipitations au-dessus du peuplement et celles atteignant le sol. Elle est exprimée en hauteur d'eau $(\mathrm{mm})$ ou en pourcentage des précipitations incidentes. L'interception nette, déduction faite de l'écoulement le long des troncs, est appelée quelquefois perte par interception.

La fraction interceptée des précipitations constitue un paramètre important du bilan hydrologique. Elle représente la première influence de la forêt sur les précipitations. De nombreuses expériences ont été entreprises pour déterminer l'importance de ce phénomène. Mais, les techniques utilisées sont très différentes, et il est très difficile de comparer les résultats.

Une des premières études qui doit être citée est celle de MAtHIEU (1866) en France. Il faut mentionner également les recherches de Fautrat (1878) et Cuif (1913). A l'étranger, d'autres travaux ont été publiés par Horton (1919). KitTrege (1948), dans son ouvrage ("Forest influences)) donne une synthèse des résultats des recherches accomplies dans ce domaine. Depuis cette date, des études ont été développées dans la plupart des pays du monde. Certains aménagistes, considérant les besoins croissants en eau dans le monde, se demandent :

— si la forêt consomme plus ou moins que d'autres types de formation végétale ;

- si la forêt résineuse consomme plus ou moins d'eau que la forêt feuillue.

Il est évident que la connaissance des influences des différentes espèces et des différents peuplements devrait permettre un aménagement plus rationnel. Ces considérations peuvent alors conduire à suivre une politique différente de celle qui ne viserait que la production maximum de bois.

Dans le but d'étudier les influences de la forêt sur le bilan des eaux d'infiltration, des recherches ont été entreprises depuis 1965. Ce sont les résultats des mesures effectuées pour évaluer l'importance des phénomènes d'interception par diverses essences qui sont exposés ici.

L'expérimentation a été réalisée à $15 \mathrm{~km}$ à l'est de Nancy, à l'Arboretum d'Amance (altitude : $250 \mathrm{~m}$, latitude : $48^{\circ} 44^{\prime} \mathrm{N}$, longitude : $06^{\circ} 14^{\prime} \mathrm{E}$ ), où il existe des peuplements feuillus et des peuplements de diverses essences résineuses. Certains de ces peuplements ont une surface suffisante pour créer l'ambiance forestière qu'aurait un peuplement étendu. Par ailleurs, ils sont suffisamment proches (situés dans un rayon de $300 \mathrm{~m}$ ) sur un terrain non accidenté, pour qu'ils puissent être considérés comme étant placés dans des conditions analogues au point de vue climatique. De plus, ces peuplements ont un âge très voisin (30 ans environ).

\section{2. - DISPOSITIF EXPÉRIMENTAL}

\section{1. - Généralités}

Les peuplements étudiés ont une structure homogène : futaie à un seul étage, équienne, sans strate arbustive (voir tableau 1 - photo 1). 


\section{TABLEAU 1}

Caractéristiques des peuplements étudiés

\begin{tabular}{|c|c|c|c|c|}
\hline Espèce & $\begin{array}{l}\text { Age } \\
\text { ans }\end{array}$ & $\begin{array}{l}\text { Hauteur } \\
\text { totale } \\
\text { moyenne }(\mathrm{m})\end{array}$ & $\begin{array}{l}\text { Nombre de } \\
\text { tiges/ha }\end{array}$ & $\begin{array}{l}\text { Diamètre }(\mathrm{cm}) \\
\text { moyen à } 1,30 \mathrm{~m}\end{array}$ \\
\hline Pinus silvestris L. .......... & 28 & 13,0 & 1520 & 17,0 \\
\hline Picea abies (L. Karsten) ...... & 30 & 12,5 & 2160 & 12,0 \\
\hline $\begin{array}{l}\text { Abies grandis (Dougl. Lindt) } \\
\quad \text { (Sapin de Vancouver) ..... }\end{array}$ & 35 & 23,0 & 620 & 30,3 \\
\hline $\begin{array}{l}\text { Peuplement feuillu .......... } \\
\text { Fagus silvatica } \mathrm{L} . \\
\text { Carpinus betulus } \mathrm{L} .\end{array}$ & 30 & 12,5 & 1300 & 8,7 \\
\hline
\end{tabular}

Le régime pluviométrique de la région est un régime de transition mi-océanique, mi-continental, avec deux maxima : en novembre et juin. Les normales mensuelles (1921-1950) des précipitations (en $\mathrm{mm}$ ) sont les suivantes à Nancy :

$\begin{array}{ccccccccccccc}\mathrm{J} & \mathrm{F} & \mathrm{M} & \mathrm{A} & \mathrm{M} & \mathrm{J} & \mathrm{J} & \mathrm{A} & \mathrm{S} & \mathrm{O} & \mathrm{N} & \mathrm{D} & \text { Année } \\ 60 & 50 & 41 & 56 & 60 & 66 & 58 & 63 & 64 & 64 & 69 & 68 & 719\end{array}$

Les observations effectuées à l'Arboretum d'Amance donnent pour les 16 dernières années une pluviométrie de $662,6 \mathrm{~mm}$, contre $700,0 \mathrm{~mm}$ à Nancy pendant la période correspondante. Il semble donc que les précipitations soient plus faibles à l'Arboretum.

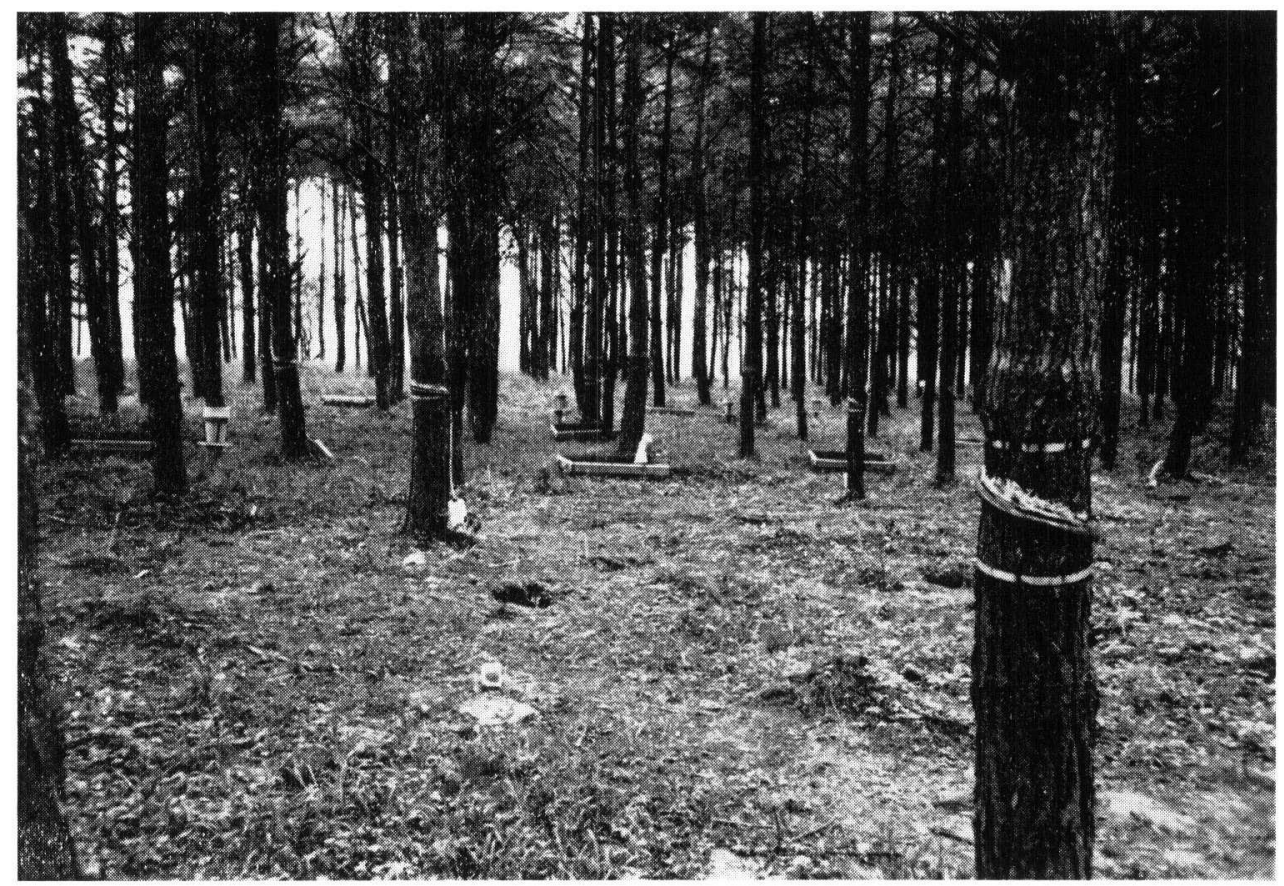

Photo 1. - Un aspect du peuplement de Pins sylvestres 


\section{2. - Mesures des précipitations tombant sur les peuplements}

Les précipitations arrivant sur les peuplements ont été évaluées à l'aide de deux pluviomètres type «association »* placés au sommet d'une tour élevée dans un peuplement. Les surfaces réceptrices des deux pluviomètres sont disposées horizontalement au niveau supérieur des couronnes (13,60 m), de manière à ce que la perturbation aérodynamique due aux deux appareils soit minimum. Cette disposition particulière a été adoptée car l'installation de pluviométres dans une clairière aurait pu donner des valeurs peu représentatives des précipitations arrivant sur les peuplements, à cause des tourbillons verticaux de vent qui peuvent augmenter les précipitations dans les zones clairiérées (GeIGER, 1965). Finalement, les précipitations ont été évaluées en faisant la moyenne des hauteurs d'eau recueillies dans les deux pluviomètres. Il faut noter que la hauteur d'eau n'est pas statistiquement différente.

A partir du mois d'avril 1966, un poste de plein découvert a été installé à $500 \mathrm{~m}$ des peuplements, la pluviométrie est légèrement plus élevée en plein découvert, mais, pendant les mois de janvier, février, mars, avril 1967, c'est le contraire. Les différences journalières ne dépassent pas 0,5 $\mathrm{mm}$. Ces écarts peuvent peut-être s'expliquer par des différences dans le coefficient de captation des pluviomètres utilisés, ceci en rapport avec la vitesse du vent et la situation plus ou moins exposée du pluviomètre.

En effet, d'après diffèrents auteurs cela se traduit par une évaluation par défaut des précipitations (10 à $15 \%$ ). Ceci est particulièrement important dans le cas qui nous occupe puisque la position et l'exposition au vent des pluviomètres utilisés sont différentes.

Dans le cas de l'évaluation des précipitations incidentes, on a donc une erreur par défaut, alors que pour l'évaluation des précipitations au sol, le coefficient de captation des pluviomètres est plus élevé.

En fin de compte, l'évaluation de l'interception se fera par défaut.

\section{3. - Mesure des précipitations arrivant directement au sol}

Pour évaluer les précipitations arrivant directement au sol, des pluviomètres « association » ont été également utilisés. Le couvert forestier présente une grande hétérogénéité et une évaluation correcte de la pluviométrie au sol est difficile. Pour essayer de résoudre ce problème, deux modalités de mesure ont été adoptées. D’une part, une série de pluviomètres ont été installés à poste fixe, après que leur emplacement ait été choisi au hasard ; d'autre part, une deuxième série était constituée par des pluviomètres " mobiles».

Au cours de l'expérience, chacun de ces pluviomètres a été déplacé sur une série de 12 points choisis au hasard sur une grille (à maille de $75 \mathrm{~cm}$ de côté) couvrant le plateau d'expérimentation. Le déplacement d'un point à un autre se fait chaque fois que $30 \mathrm{~mm}$ sont tombés sur les peuplements (le changement de position s'effectuant simultanément dans les 4 peuplements étudiés). WICHT (1941) utilisait un système de pluviomètres mobiles qui étaient déplacés sur les côtés d'un carré. utilisés.

Le tableau 2 donne pour chaque essence le nombre et les caractéristiques des pluviomètres

\section{TABLEAU 2}

Nombre de pluviomètres utilisés dans chaque peuplement.

\begin{tabular}{|c|c|c|c|}
\hline Peuplement & Pluviomètres fixes & Pluviomètres mobiles & Total \\
\hline Pinus silvestris $\ldots \ldots \ldots \ldots \ldots$ & 6 & 6 & 12 \\
\hline Picea abies .............. & 4 & 4 & 8 \\
\hline Abies grandis $\ldots \ldots \ldots \ldots \ldots \ldots$ & 4 & 4 & 8 \\
\hline Peuplement feuillu $\quad \ldots \ldots \ldots$ & 6 & 6 & 12 \\
\hline
\end{tabular}

* Ce type de pluviomètre a été mis au point en 1867 par l'Association scientifique de France, d'où le nom qui a été retenu aujourd'hui. La surface réceptrice est de $400 \mathrm{~cm}^{2}$ avec un diamètre de $22,6 \mathrm{~cm}$. 
Les précipitations arrivant au sol sont évaluées en effectuant la moyenne des hauteurs d'eau recueillie dans chaque pluviomètre.

\section{4. - Mesure d'écoulement le long des troncs}

L'eau qui s'écoule le long des troncs peut représenter une fraction non négligeable de l'eau retournant au sol, ce qui peut constituer une modification importante du bilan hydrologique autour des troncs. Pour l'évaluer, nous avons été amené à concevoir un système de collier en matière plastique (photo 2). Il s'agit d'un dispositif entourant entièrement le tronc à une hauteur de $80 \mathrm{~cm}$. L'étanchéité est assurée par un glacis de mastic à greffer. La fixation est réalisée par un dispositif constitué par une tige filetée qui permet de desserrer le collier au fur et à mesure de la croissance de l'aróre. Les colliers installés sont au nombre de 8 pour les Pins sylvestres, 6 pour les Epicéas, 5 pour les Sapins de Vancouver et 14 pour le peuplement feuillu.

L'eau recueillie est stockée dans des bidons au pied du tronc. Les arbres de chaque placeau ont été ventilés dans différentes classes de diamètre, ce qui a permis d'installer les colliers en fonction de cette répartition. Dans chaque classe, la désignation des arbres échantillonnés a été faite au hasard.

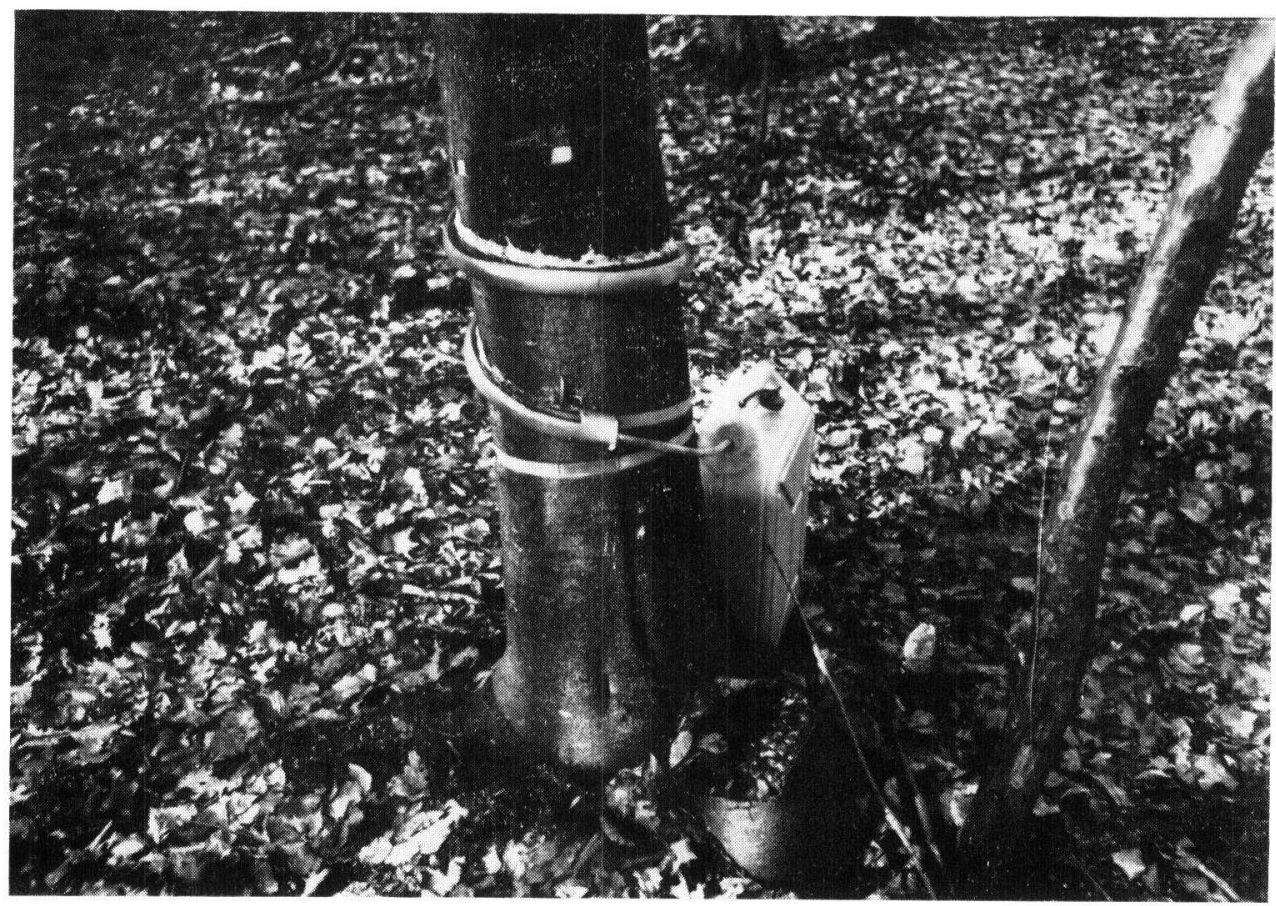

Photo 2. - Coilier collecteur sur hêtre

Certains auteurs ont trouvé une relation linéaire entre les quantités d'eau récoltées et la circonférence du tronc à $1,30 \mathrm{~m}$. Cette relation n'a pas été retrouvée dans les peuplements étudiés. Les classes de diamètre n'étaient pas suffisamment nombreuses, peut-on penser.

Le volume total d'eau arrivant au sol est calculé en multipliant le volume d'eau recueillie par les arbres échantillonnés par le rapport du nombre total d'arbres dans le placeau au nombre d'arbres échantillons. Ce volume est ensuite transformé en hauteur d'eau.

Les mesures sont effectuées de façon journalière dans toute le dispositif. L'interception nette est calculée par la formule :

$$
I_{n}=P_{I}-\left(P_{S}+S\right)
$$


où $P_{I}$ représente la pluviométrie en $\mathrm{mm}$ au-dessus des peuplements, $P_{S}$ la pluviométrie au sol et $S$ l'écoulement le long des troncs.

Les mesures ont commencé au 1-4-65 pour les résineux et au 7-7-66 pour les feuillus.

\section{3. - RÉSULTATS}

\section{1. - Estimation de l'interception}

Le calcul de la fraction interceptée, c'est-à-dire l'interception nette, déduction faite de l'eau s'écoulant le long des troncs, a été effectué pour chaque jour de pluie. Les tableaux 3 - 4 et 5 donnent mensuellement pour les différents peuplements étudiés : l'estimation des précipitations arrivant directement au sol $\left(P_{S}\right)$, l'écoulement le long des troncs $(S)$ et l'interception nette $\left(I_{n}\right)$. D'une façon générale, les peuplements résineux interceptent beaucoup plus que le peuplement feuillu.

\section{TABLEAU 3}

Estimation de la pluviométrie, de l'eau au sol, de l'écoulement le long des troncs et de l'interception nette pour les peuplements de Pin sylvestre et Epicéa

\begin{tabular}{|c|c|c|c|c|c|c|c|c|c|c|c|c|c|}
\hline \multirow{3}{*}{ Mois } & \multirow{3}{*}{$\begin{array}{c}\text { Pluie } \\
\text { inci- } \\
\text { dente } \\
\mathrm{mm} \\
P_{I}\end{array}$} & \multicolumn{6}{|c|}{ Pin sylvestre } & \multicolumn{6}{|c|}{ Epicéa } \\
\hline & & \multicolumn{2}{|c|}{$\begin{array}{c}\text { Eau au sol } \\
P_{S}\end{array}$} & \multicolumn{2}{|c|}{$\begin{array}{c}\text { Ecoulement } \\
\text { tronc } \\
S\end{array}$} & \multicolumn{2}{|c|}{$\begin{array}{c}\text { Interception } \\
\text { nette } \\
I_{n}\end{array}$} & \multicolumn{2}{|c|}{$\begin{array}{c}\text { Eau au sol } \\
P_{S}\end{array}$} & \multicolumn{2}{|c|}{$\begin{array}{c}\text { Écoulement } \\
\text { trone } \\
S\end{array}$} & \multicolumn{2}{|c|}{$\begin{array}{c}\text { Interception } \\
\text { nette } \\
I_{n}\end{array}$} \\
\hline & & $\mathrm{mm}$ & $\%$ & $\mathrm{~mm}$ & $\%$ & $\mathrm{~mm}$ & $\%$ & $\mathrm{~mm}$ & $\%$ & $\mathrm{~mm}$ & $\%$ & $\mathrm{~mm}$ & $\%$ \\
\hline Mai 1965 & 77,8 & 54,3 & 69,8 & 0 & 0,8 & 22,9 & 29,4 & 44,1 & 56,7 & 1,0 & 1,2 & 32,7 & 42,1 \\
\hline Juin . & 77,0 & 56,1 & 72,9 & 0 , & 1, & 2 & & 57,7 & & 1,0 & & 18,3 & \\
\hline Juillet & 50,3 & 32,2 & 63,9 & 0 , & 0,3 & $1 \varepsilon$ & 3 & 29,5 & 58,7 & 0,3 & 0 & 20,5 & \\
\hline Août & 27,8 & 16 & 58 & 0 , & 0 & & & 11,2 & 40,3 & 0,1 & 0 & 16,6 & \\
\hline Septembre & 107,3 & 74,4 & 69 , & 1 , & 1 , & & & & 75,9 & 2,1 & 1,9 & 23,8 & \\
\hline Octobre . . & 6,2 & 2,7 & 43 , & 0, & 0, & & & 2,5 & 3 & 0,0 & 0,0 & 3,7 & 59,7 \\
\hline Novembre & 108,6 & 76,1 & 70 & 2 , & 2 , & & 2 & 73,4 & & 2,8 & & 32,4 & \\
\hline Décembre. & 145,7 & 105 , & 72 & 4, & 2 , & & & & & 5,0 & & 43,5 & \\
\hline Janvier 1966 & 78,1 & 54 & 70 & 1 , & 1 , & 2. & & 49 & & 1 , & & 26,7 & \\
\hline Février ..... & 63,3 & 41, & 66 & 2 , & 3, & & & 45 & 72 & 1 . & & 16,0 & J \\
\hline Mars ...... & 53,1 & 23 , & 43 & 0 & 1 & & & 21 & & 0 & 0 & 31,6 & \\
\hline Avril & 87,2 & 55, & 64,1 & 1 , & 1 & & & 47, & 54,8 & 1,2 & 1,4 & 38,1 & 8 \\
\hline Année ... & 882,4 & 592,7 & 67,2 & 15,0 & 1,7 & 274,7 & 31,1 & 561,5 & 63,6 & 16,9 & & 304,0 & \\
\hline Mai 1966 & 56,7 & 39,4 & 69,4 & 0. & 0 & 16.8 & 2 & 32,1 & 56,5 & 0,3 & 0.6 & 24,3 & 2,9 \\
\hline & 60,8 & 44,2 & 72,7 & 0 , & 0 & & & & & 0 & & 9,7 & \\
\hline $\mathrm{Ju}$ & 50,2 & 32,3 & 64,4 & 0 , & 0 & & & 2 & 8 & 0 & 0 & 25,9 & \\
\hline Août & 94,6 & 68 , & 72 & 1, & 1 , & & & & & 0 & & 18,7 & \\
\hline Septen & 42,7 & 29, & 68 & 0 , & 1 & & & & & 0 & & 12,3 & \\
\hline Octobre . . & 77,3 & 55,2 & 71 & 1, & 1 , & & & 45,2 & & & & 30,1 & \\
\hline Novembre & 58,0 & 39 , & 68 & 1 , & & & & & & 1 & & 17,3 & 8 \\
\hline Décembre. . & 158,0 & 118,5 & 75 & 3, & 2 & 36 & 22,9 & 107,3 & 67,9 & 4 & & 45,9 & \\
\hline Janvier 1967 & 33,7 & 23,1 & 68 & 0 , & 1 & & & & & 0 & & 7,2 & 2 \\
\hline Février ... & 41,6 & 18,9 & 45 & 0 , & 1, & & & 15 & 37,0 & 0 & & 25,7 & \\
\hline Mars . & 45,8 & 24,0 & 52,5 & 0 & 1 , & & & 20 & & 0 , & & 24,4 & 53,2 \\
\hline Avril & 31,1 & 17,7 & 56,8 & 0, & 1,0 & & & 15 , & 48,4 & 0,3 & 1,0 & 15,7 & 50,6 \\
\hline Année & 750,5 & 510,2 & 68,0 & 11,4 & 1,5 & 228,9 & 30,5 & 479,9 & 63,9 & 13,4 & 1,8 & 257,2 & 34,3 \\
\hline
\end{tabular}




\section{TABLEAU 4}

Estimation de la pluviométrie, de l'eau au sol, de l'écoulement le long des troncs et de l'interception nette pour le peuplement de Sapin de Vancouver

\begin{tabular}{|c|c|c|c|c|c|c|c|}
\hline \multirow[t]{2}{*}{ Mois } & \multirow{2}{*}{$\begin{array}{l}\text { Pluie } \\
\text { inci- } \\
\text { dente } \\
\text { mm } \\
P_{I}\end{array}$} & \multicolumn{2}{|c|}{$\begin{array}{c}\text { Eau au sol } \\
P_{S}\end{array}$} & \multicolumn{2}{|c|}{$\begin{array}{c}\text { Ecoulement } \\
\text { tronc } \\
S\end{array}$} & \multicolumn{2}{|c|}{$\begin{array}{c}\text { Interception } \\
\text { nette } \\
I_{n}\end{array}$} \\
\hline & & $\mathrm{mm}$ & $\%$ & $\mathrm{~mm}$ & $\%$ & $\mathrm{~mm}$ & $\%$ \\
\hline Mai $1965 \ldots \ldots \ldots \ldots \ldots$ & 77,8 & 51,6 & 66,3 & 0,6 & 0,7 & 25,7 & 33,0 \\
\hline Juin $\ldots \ldots \ldots \ldots \ldots \ldots$ & 77,0 & 53,2 & 69,1 & 0,9 & 1,1 & 22,9 & 29,8 \\
\hline Juillet $\quad \ldots \ldots \ldots \ldots \ldots$ & 50,3 & 19,1 & 38,0 & 0,2 & 0,4 & 31,0 & 61,6 \\
\hline Août $\ldots \ldots \ldots \ldots \ldots \ldots$ & 27,8 & 10,7 & 38,5 & 0,1 & 0,3 & 17,0 & 61.2 \\
\hline Septembre $\quad \ldots \ldots \ldots \ldots$ & 107,3 & 60,9 & 56,8 & 1,3 & 1,2 & 45,1 & 42,0 \\
\hline Octobre $\ldots \ldots \ldots \ldots \ldots$ & 6,2 & 1,2 & 19,3 & 0,0 & 0,0 & 5,0 & 80,7 \\
\hline Novembre $\quad \ldots \ldots \ldots \ldots$ & 108,6 & 62,9 & 57,9 & 1,7 & 1,6 & 44,0 & 40,5 \\
\hline Décembre $1965 \ldots \ldots \ldots \ldots$ & 145,7 & 88,0 & 60,4 & 2,3 & 1,6 & 55,4 & 38,0 \\
\hline Janvier $1966 \ldots \ldots \ldots \ldots$ & 78,1 & 43,4 & 55,6 & 0,7 & 0,8 & 34,0 & 43,6 \\
\hline Février $\ldots \ldots \ldots \ldots \ldots$ & 63,3 & 32,5 & 51,3 & 0,9 & 1,4 & 29,9 & 47,3 \\
\hline Mars $\ldots \ldots \ldots \ldots \ldots$ & 53,1 & 16,5 & 31,1 & 0,3 & 0,6 & 36,2 & 68,3 \\
\hline Avril $\ldots \ldots \ldots \ldots \ldots$ & 87,2 & 52,1 & 59,8 & 0,5 & 0,6 & 34,5 & 39,6 \\
\hline Année $\quad \ldots \ldots \ldots \ldots \ldots$ & 882,4 & 492,1 & 55,8 & 9,5 & 1,1 & 380,7 & 43,1 \\
\hline Mai $1966 \ldots \ldots \ldots \ldots \ldots$ & 56,7 & 34,2 & 60,3 & 0,4 & 0,8 & 22,0 & 38,9 \\
\hline Juin $\ldots \ldots \ldots \ldots \ldots$ & 60,8 & 29,9 & 49,2 & 0,4 & 0,7 & 30,5 & 50,1 \\
\hline Juillet $\quad \ldots \ldots \ldots \ldots \ldots$ & 50,2 & 29,0 & 57,7 & 0,3 & 0,5 & 21,0 & 41,8 \\
\hline Août $\ldots \ldots \ldots \ldots \ldots$ & 94,6 & 62,6 & 66,2 & 0,6 & 0,6 & 31,4 & 33,2 \\
\hline Septembre . . . . . . . . . & 42,7 & 28,0 & 65,6 & 0,6 & 1,5 & 14,1 & 32,9 \\
\hline Octobre $\ldots \ldots \ldots \ldots \ldots$ & 77,3 & 53,2 & 68,8 & 0,6 & 0,8 & 32,5 & 30,4 \\
\hline Novembre $\quad \ldots \ldots \ldots \ldots$ & 58,0 & 25,3 & 43,6 & 0,8 & 1,4 & 31,9 & 55,0 \\
\hline Décembre $\ldots \ldots \ldots \ldots \ldots$ & 158,0 & 107,4 & 68,0 & 2,1 & 1,3 & 48,5 & 30,7 \\
\hline Janvier $1967 \quad \ldots \ldots \ldots \ldots$ & 33,7 & 18,3 & 54,2 & 0,4 & 1,2 & 15,0 & 44,6 \\
\hline Février $\ldots \ldots \ldots \ldots \ldots$ & 41,6 & 10,9 & 26,2 & 0,4 & 0,9 & 30,3 & 72,9 \\
\hline Mars $\ldots \ldots \ldots \ldots \ldots$ & 45,8 & 17,4 & 37,9 & 0,3 & 0,7 & 28,1 & 61,4 \\
\hline Avril $\ldots \ldots \ldots \ldots \ldots$ & 31,1 & 17,4 & 56,0 & 0,2 & 0,7 & 13,5 & 43,3 \\
\hline Année $\ldots \ldots \ldots \ldots \ldots$ & 750,5 & 433,6 & 57,8 & 7,1 & 1,0 & 309,8 & 41,2 \\
\hline
\end{tabular}

Pour un bilan annuel on trouve :

- le peuplement de Sapin de Vancouver (Abies grandis) avec, pour les deux années de mesure, un pourcentage d'interception nette supérieur à $40 \%(43,2 \%$ - 1965-1966 et 41,6\% - 1966-1967),

- le peuplement d'Epicéa (Picea abies) $34 \%(34,4 \%$ - 1965-1966 - et 34,3\%-1966-1967),

- les Pins sylvestres (Pinus silvestris) avec un pourcentage voisin de $31 \%$ $(31,6 \%-1965-1966$ et 30,5\% (1966-1967),

- le peuplement feuillu (Fagus silvatica, Carpinus betulus) $16,9 \%$. Il y a une différence entre les périodes feuillées $(18,6 \%)$ et défeuillées $(15,1 \%)$.

Comme nous l'avons dit précédemment, l'évaluation des précipitations incidentes est faite par défaut alors que l'évaluation des précipitations au sol est relativement précise. En fin de compte, cela se traduit par une sous-estimation de l'importance de l'interception. 


\section{Tableau 5}

Estimation de l'eau au sol, de l'écoulement le long des troncs et de l'interception nette pour le peuplement feuillu

\begin{tabular}{|c|c|c|c|c|c|c|c|}
\hline \multirow[t]{2}{*}{ Mois } & \multirow{2}{*}{$\begin{array}{l}\text { Pluvio- } \\
\text { métrie } \\
\quad P_{I}\end{array}$} & \multicolumn{2}{|c|}{$\begin{array}{c}\text { Eau aul sol } \\
P_{S}\end{array}$} & \multicolumn{2}{|c|}{$\begin{array}{c}\text { Ecoulement } \\
\text { tronc } \\
\mathrm{S}\end{array}$} & \multicolumn{2}{|c|}{$\begin{array}{c}\text { Interception } \\
\text { nette } \\
I_{n}\end{array}$} \\
\hline & & $\mathrm{mm}$ & $\%$ & $\mathrm{~mm}$ & $\%$ & $\mathrm{~mm}$ & $\%$ \\
\hline Juillet $1966 \ldots \ldots \ldots \ldots$ & 24,2 & 17,7 & 73,2 & 1.7 & 7,0 & 4.8 & 19.8 \\
\hline Août $\ldots \ldots \ldots \ldots \ldots \ldots$ & 94,6 & 72,6 & 76,8 & 5,3 & 5,6 & 16,7 & 17,6 \\
\hline Septembre $\quad \ldots \ldots \ldots \ldots$ & 42,7 & 30,4 & 71,2 & 3,7 & 8,7 & 8,6 & 20,1 \\
\hline 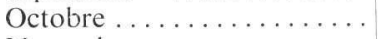 & 77,3 & 60,0 & 77,6 & 4,8 & 6,2 & 12,5 & 16,2 \\
\hline Novembre $\ldots \ldots \ldots \ldots \ldots$ & 58,0 & 46,1 & 79,5 & 5,3 & 9,2 & 6,6 & 11,3 \\
\hline Décembre $\ldots \ldots \ldots \ldots$ & 158,0 & 118,1 & 74,8 & 11,0 & 6,9 & 28,9 & 18,3 \\
\hline Janvier $1967 \ldots \ldots \ldots$ & 33,7 & 29,1 & 86,5 & 2,2 & 6,5 & 2,4 & 7,0 \\
\hline 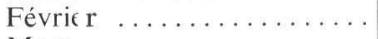 & 41,6 & 31,9 & 76,7 & 3,3 & 8,1 & 6,3 & 15,2 \\
\hline 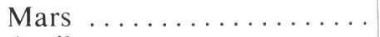 & 45,8 & 35,1 & 76,6 & 4,1 & 9,0 & 6,6 & 14,4 \\
\hline Avril $\ldots \ldots \ldots \ldots \ldots$ & 31,1 & 23,8 & 76,4 & 2,6 & 8,2 & 4,8 & 15,4 \\
\hline 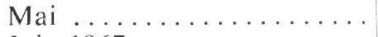 & 75,8 & 53,2 & 70,3 & 5,0 & 6,6 & 17,5 & 23,1 \\
\hline Juin $1967 \ldots \ldots \ldots \ldots$ & 41,4 & 32,4 & 78,3 & 2,0 & 4,9 & 7,0 & 16,8 \\
\hline Annźe $\ldots \ldots \ldots \ldots$ & 724,2 & 550,4 & 76,0 & 51,0 & 7,1 & 122,7 & 16,9 \\
\hline
\end{tabular}

L'écoulement le long des troncs est faible pour les résineux :

- Epicéas : 1,9\% (1965-1966), 1,8\% (1966-1967),

— Pins sylvestres : 1,7\% (1965-1966), 1,5\% (1966-1967),

- Sapins de Vancouver : 1,0\% (1965-1965), 1,0\% (1966-1967).

Dans le peuplement feuillu, l'écoulement est important, et sur l'année il atteint $7 \%$ des précipitations incidentes. Pendant la période défeuillée, les pourcentages sont plus élevés $(7,7 \%$ ) que pendant la phase de végétation $(6,3 \%)$. Ces écoulements élevés peuvent être attribués à la morphologie particulière des feuillus et du hêtre en particulier. En effet, l'insertion des branches sur le tronc se fait avec un angle aigu et toute l'eau s'écoulant le long des branches est ramenée sur les trones.

Il faut remarquer que l'incidence de l'écoulement le long des troncs est beaucoup plus grande que ce que l'on peut voir de prime abord. En effet, même dans le cas des Pins sylvestres ou des Sapins de Vancouver, ce phénomène se traduit par un apport important d'eau aux alentours immédiats des troncs.

Pour des précipitations très faibles, l'interception est totale $(100 \%)$. L'analyse des pluies journalières montre que toutes les précipitations sont totalement interceptées au-dessous des hauteurs de pluie suivantes :

- 0,6 mm pour les Epicéas,

- $0.5 \mathrm{~mm}$ pour les Sapins de Vancouver,

- 0,4 mm pour les Pins sylvestres,

- $0,3 \mathrm{~mm}$ pour le peuplement feuillu. 
L'écoulement le long des troncs commence à se manifester dès $0,9 \mathrm{~mm}$ dans le peuplement feuillu ; pour les résineux, il faut $1,5 \mathrm{~mm}$ chez les sapins de Vancouver, $1,3 \mathrm{~mm}$, chez les Pins sylvestres et les Epicéas. L'écoulement augmente avec l'intensité des précipitations.

\section{2. - La capacité de saturation des couronnes.}

La capacité de saturation des couronnes des peuplements est un paramètre intéressant à connaître. La hauteur d'eau maximum susceptible d'être retenue est une fonction de la surface foliaire et de la surface des branches et rameaux, de la morphologie des feuilles et du type de ramification. Au début d'une averse, la plupart des gouttes d'eau sont interceptées par les houppiers. L'eau s'étend alors sur les surfaces végétales et s'accumule jusqu'à ce que la capacité de stockage maximum soit atteinte, c'est-à-dire jusqu'à ce que les forces de tension de surface soient dépassées par la force de pesanteur. Les phénomènes d'évaporation interviennent au début, car les tempśratures de surface des feuilles peuvent être plus élevées que celles de l'air ; mais avec le développement de l'averse, ces phénomènes diminuent rapidement. Finalement, quand la capacité de saturation est atteinte, les couronnes ne retiennent plus que les quantités additionnelles nécessaires à la compensation de l'eau évaporée. Si l'averse continue, les peuplements n'interceptent pratiquement plus. Le couvert n'a alors qu'un rôle mécanique.

Pour évaluer cette capacité de saturation maximum, nous avons recherché les averses importantes qui n'ont pas présenté d'interruption. Cela a pu être fait grâce à un pluviographe installé en plein découvert (à $500 \mathrm{~m}$ des peuplements). Ces averses sont tombées pendant la nuit et les phénomènes d'évaporation ont été faibles. Le tableau 6 donne pour ces averses types : les précipitations sur les peuplements et l'interception. Les valeurs obtenues sont les suivantes:

- Sapin de Vancouver : $3,8 \pm 1,5 \mathrm{~mm}$,

— Epicéas $\quad: 3,1 \pm 0,8 \mathrm{~mm}$,

- Pins sylvestres $\quad: 3,0 \pm 0,8 \mathrm{~mm}^{*}$,

— peuplement feuillu : $1,9 \pm 0,5 \mathrm{~mm}$.

TABleau 6

Interception (en mm) pour des averses continues

\begin{tabular}{|c|c|c|c|c|c|}
\hline \multirow[b]{2}{*}{ Date } & \multirow{2}{*}{$\begin{array}{c}\text { Précipitations } \\
(\mathrm{mm})\end{array}$} & \multicolumn{4}{|c|}{ Interception } \\
\hline & & $\begin{array}{r}\text { Pinus } \\
\text { silvestris }\end{array}$ & $\begin{array}{l}\text { Picea } \\
\text { abies }\end{array}$ & $\begin{array}{c}\text { Abies } \\
\text { grandis }\end{array}$ & Feuillus \\
\hline 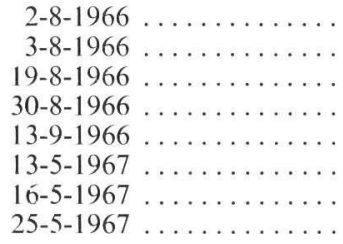 & $\begin{array}{r}11,5 \\
13,0 \\
32,5 \\
9,1 \\
5,2 \\
7,4 \\
11,0 \\
6,2\end{array}$ & $\begin{array}{l}2,6 \\
3,6 \\
3,9 \\
2,8 \\
2,4\end{array}$ & $\begin{array}{l}2,9 \\
3,9 \\
2,9 \\
2,8\end{array}$ & $\begin{array}{l}2,7 \\
4,3 \\
4,8 \\
3,4 \\
-\end{array}$ & $\begin{array}{l}1,6 \\
2,8 \\
1,2 \\
1,6 \\
2,3 \\
1,6 \\
2,5\end{array}$ \\
\hline
\end{tabular}

* Intervalle de confiance à $P=0,95$. 
L'examen des résultats obtenus montre que pour une même hauteur d'eau tombant sur les peuplements, l'interception est beaucoup plus importante si la pluie tombe en petites averses entrecoupées de périodes de temps clair (tableau 7). En effet, pendant la période sans pluie, l'eau interceptée s'évapore et le (" réservoir ) couronnes est à nouveau disponible pour se recharger et ainsi de suite.

TABLEAU 7

Interception $(\mathrm{mm})$ pour des averses discontinues

\begin{tabular}{|c|c|c|c|c|c|}
\hline & Date & $\begin{array}{l}\text { Précipitations } \\
(\mathrm{mm})\end{array}$ & $\begin{array}{c}\text { Pins } \\
\text { sylvestres }\end{array}$ & Epicéa & $\begin{array}{l}\text { Sapin de } \\
\text { Vancouver }\end{array}$ \\
\hline $\begin{array}{l}6-9-1965 \\
1-10-1965 \\
6-12-1965 \\
5-7-1966\end{array}$ & 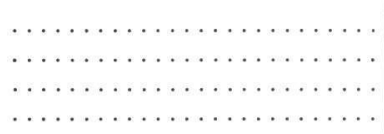 & $\begin{array}{l}17,8 \\
22,4 \\
21,9 \\
18,4\end{array}$ & $\begin{array}{l}5,5 \\
4,3 \\
4,8 \\
5,1\end{array}$ & $\begin{array}{l}6,2 \\
4,5 \\
5,0 \\
6,0\end{array}$ & $\begin{array}{l}6,8 \\
7,4 \\
8,0 \\
5,2\end{array}$ \\
\hline
\end{tabular}

3.3. - Explication des précipitations arrivant directement au sol en fonction des précipitations incidentes et de l'éclairement relatif.

L'interception dépend de la surface foliaire et de la morphologie des rameaux et des branches (photo 3 ). Il est très difficile d'évaluer ces paramètres par des mesures directes : projection des couronnes, épaisseurs de la voûte foliacée... Pour essayer de tourner cette difficulté, nous avons utilisé une méthode reposant sur la mesure de l'éclairement relatif. Ces mesures ont été réalisées au-dessus de chaque emplacement de pluviomètres, à l'aide d'une cellule photoélectrique. L'éclairement relatif dépend toutes choses égales par ailleurs, de l'épaisseur de la couronne, de la disposition des feuilles et des branches, et de la densité de la voûte foliacée. L'échantillonnage a porté sur la portion de couronne se trouvant directement au-dessus des surfaces réceptrices des pluviomètres. Dans ce but, nous avons été conduit à réaliser un appareillage particulier constitué par une cellule photorésistante au Sulfure de Cadmiun (Métrastar) placée à l'extrémité d'un tube de $100 \mathrm{~cm}$ de long et de $5 \mathrm{~cm}$ de diamètre $\left({ }^{1}\right)$. De cette manière, l'angle de mesure est extrêmement réduit $\left(2^{\circ} 51^{\prime}\right)$.

Lorsque l'éclairement augmente en plein découvert, l'éclairement relatif $(\%)$ dans le peuplement diminue. Cependant NaEgel (1940) et Roussel (1953) ont montré que par temps couvert bien égal, sans vent et nuages élevés, la valeur de l'éclairement relatif reste relativement constante, quel que soit l'éclairement extérieur. Ce type de temps a été choisi pour effectuer les mesures.

De cette façon, on a pu effectuer un échantillonnage de l'éclairement relatif dans les peuplements. Il a porté sur 78 points dans les Pins sylvestres et le peuplement feuillu, 52 points dans les Epicéas et les Sapins de Vancouver.

A la suite de ces mesures, une étude a été entreprise pour essayer de lier les précipitations arrivant directement au sol en un point déterminé aux précipitations

(1) En fait, ce qui est mesuré ici, c'est l'opacité optique du feuillage à la verticale du point de mesure. Le terme d'éclairement relatif n'est donc pas absolument exact et ne doit pas être pris dans le sens de l'éclairement au sol du point de mesure. 


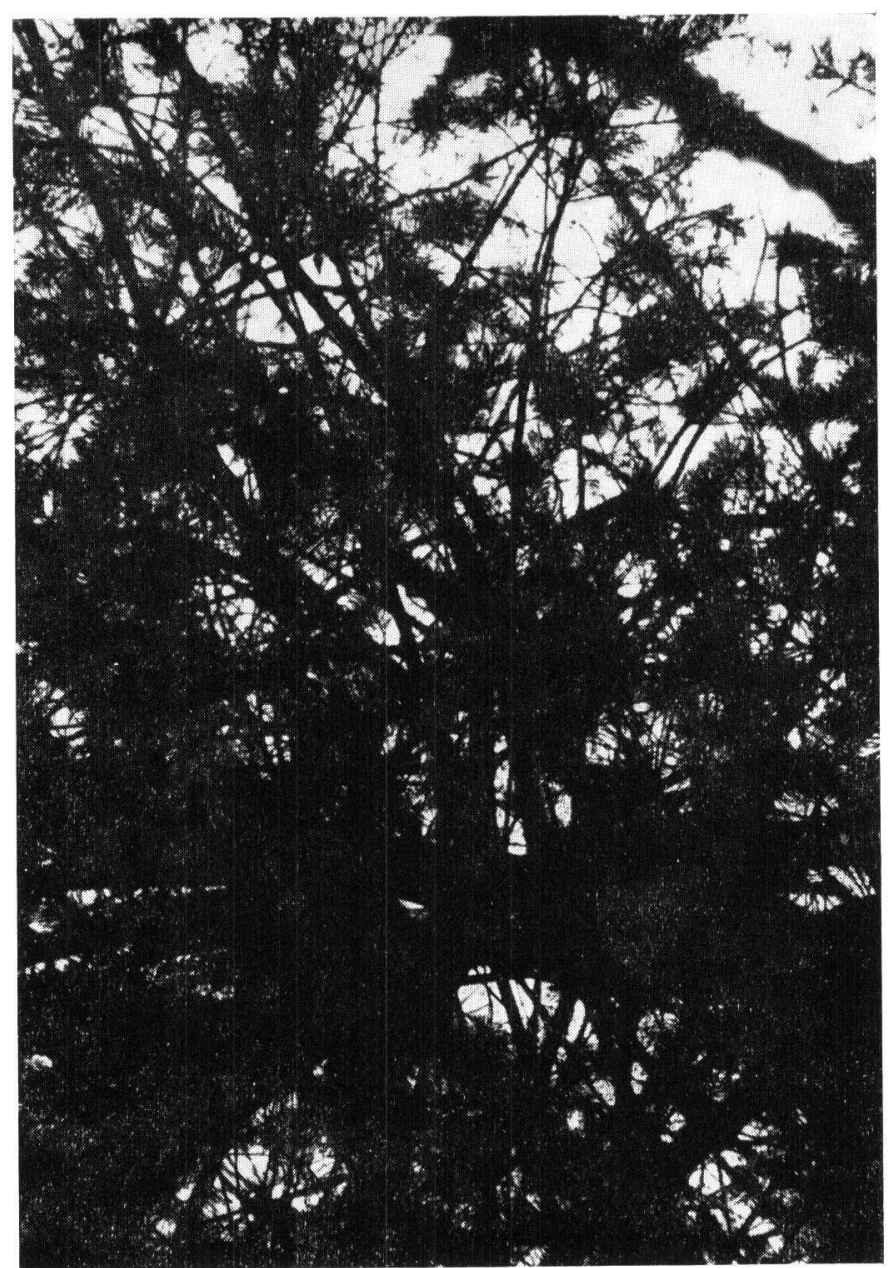

Photo 3. - Aspect du couvert dans le peuplement de Pins sylvestres

incidentes et à l'éclairement relatif. Les calculs de régression progressive ont été effectués par C. Millier, de la Station de Biométrie du C.N.R.F. Il s'agit d'une méthode d'explication d'une variable $Y$ en fonction de $p$ variables explicatives. Cette méthode donne les équations successives qui ajustent le mieux $Y$. A chaque palier du calcul, le programme donne :

- l'écart type résiduel,

- le coefficient de corrélation multiple $R^{2}$,

- le terme constant $\left(b_{0}\right)$,

- les coefficients de l'équation de régression :

$b_{1}$ : éclairement relatif $E$,

$b_{2}$ : pluie incidente $X$,

$b_{3}$ : pluie incidente au carré $X^{2}$. 
Pour obtenir les meilleures équations, une transformation logarithmique a été nécessaire. Les équations sont alors de la forme :

$$
\operatorname{LN}\left({ }^{2}\right)(Y+1)=b_{0}+b_{1} E+b_{2} X+b_{3} X^{2}
$$

Afin d'étudier en détail la relation, les calculs ont été effectués pour des périodes de 3 mois pour la première année de mesure $(01=$ mai, juin, juillet $1965 ; 02=$ août, septembre, octobre $1965 ; 03=$ novembre, décembre, janvier $1966 ; 04=$ février, mars, avril 1966 pour les résineux ; $01=$ juillet, août, septembre $1966 ; 02=$ octobre, novembre, décembre $1966 ; 03=$ janvier, février, mars $1967 ; 04=$ avril, mai, juin 1967 pour le peuplement feuillu).

Les résultats sont rapportés dans le no 8 . On constate que les relations sont très voisines d'une période à une autre pour une même espèce. Il s'agit donc d'une relation caractéristique de chaque type de peuplement.

Pour l'année, les équations sont les suivantes :

- Peuplement de Pins sylvestres :

$$
\begin{gathered}
\mathrm{LN}(Y+1)=0,0047+0,0056 E+0,2584 X-0,0063 X^{2} \\
R^{2}=0,954 \quad s=0,239
\end{gathered}
$$

- Peuplement d'Epicéas :

$$
\begin{gathered}
\mathrm{LN}(Y+1)=-0,1242+0,00795 E+0,2573 X-0,0058 X^{2} \\
R^{2}=0,919 \quad s=0,324
\end{gathered}
$$

- Peuplement de Sapins de Vancouver :

$$
\begin{gathered}
\operatorname{LN}(Y+1)=-0,0162+0,00325 E+0,2254 X-0,045 X^{2} \\
R^{2}=0,919 \quad s=0,349
\end{gathered}
$$

Les figures 1 à 4 donnent l'allure des courbes pour la période novembre-décembre 1965, janvier 1966, dans les peuplements résineux et pour la période janvier-févriermars 1967 dans le peuplement feuillu. Ces figures représentent les courbes obtenues pour trois valeurs de l'éclairement relatif (éclairement relatif faible, éclairement relatif moyen, éclairement relatif fort). Les précipitations au sol augmentent avec l'éclairement relatif. Pour des valeurs élevées de ce dernier, elles peuvent être supérieures à la pluie incidente. Ceci est dû aux phénomènes de ( gouttière ) qui sont surtout très importants dans le peuplement d'Epicéas, en raison de la morphologie particulière de cette essence (rameaux retombants).

Si l'on étudie la variation de l'interception en fonction de la pluie incidente (différence pour chaque valeur de la pluie, entre chaque point de la courbe et chaque point correspondant de la bissectrice des axes des coordonnées), on constate que 


\section{TABLEAU 8}

Résultats de l'analyse de régression progressive

\begin{tabular}{|c|c|c|c|c|c|c|c|c|c|}
\hline \multicolumn{5}{|c|}{ Pinus silvestris } & \multicolumn{5}{|c|}{ Picea abies } \\
\hline & 01 & 02 & 03 & 04 & & 01 & 02 & 03 & 04 \\
\hline$b_{0} \ldots \ldots$ & $-0,1723$ & $-0,0165$ & 0,1990 & 0,0085 & $b_{0} \ldots$ & $-0,2623$ & $-0,1059$ & $-0,0220$ & $-0,1057$ \\
\hline$b_{1} \ldots \ldots$ & $0,0087^{* *}$ & $0,0069^{* *}$ & $0,0020 * *$ & $0,0049^{* *}$ & $b_{1} \ldots$ & $0,0172 * *$ & $0,0031 *$ & $0,0041^{* *}$ & $0,0074^{* * *}$ \\
\hline$b_{2} \ldots \ldots$ & $0,2999 * *$ & $0,2673^{* *}$ & $0,2281 * *$ & $0,2383 * *$ & $b_{2} \ldots \ldots$ & $0,269+* *$ & $0,2791 * *$ & $0,2398^{* * *}$ & $0,2412^{* *}$ \\
\hline$b_{3} \ldots \ldots$ & $-0,0088^{* *}$ & $-0, \cos 5^{* *}$ & $-0,0045^{* *}$ & $-0,0054^{* *}$ & $b_{3} \ldots \ldots \ldots$ & $-0,0065^{* *}$ & $-0,0068 \%$ & $-0,0046^{* *}$ & $-0,0045^{* *}$ \\
\hline$s \ldots \ldots$ & 0,272 & 0,176 & 0,248 & 0,260 & $s \ldots$ & 0,392 & 0,326 & 0,320 & 0,358 \\
\hline$R_{2} \ldots \ldots$ & 0,930 & 0,970 & 0,966 & 0,942 & $R_{2} \quad \ldots \ldots$ & 0,888 & 0,928 & 0,946 & 0,915 \\
\hline \multicolumn{5}{|c|}{ Abies grandis } & \multicolumn{5}{|c|}{ Peuplement feuillu } \\
\hline & 01 & 02 & 03 & 04 & & 01 & 02 & 03 & 04 \\
\hline$b_{0} \ldots \ldots$ & 0,1002 & $-0,1126$ & 0,0370 & $-0,0895$ & $b_{0} \quad \ldots \ldots$ & 0,1437 & 0,2487 & 0,2772 & 0,0743 \\
\hline$b_{1}$. & $0,0055^{* *}$ & $0,0024 * *$ & $0,0025 * *$ & $0,0026^{* * *}$ & $b_{1} \ldots \ldots \ldots$ & 0,0006 N.S. & $0.0027^{* *}$ & $0,0020 * *$ & $0,0027 * *$ \\
\hline$b_{2} \quad \ldots \ldots$ & $0,2055^{* *}$ & $0,2617^{* *}$ & $0,2235 * *$ & $0,2108 * *$ & $b_{2} \ldots \ldots$ & $0,2549 * *$ & $0,1958 * *$ & $0,2347 * *$ & $0,2305^{* *}$ \\
\hline$b_{3} \ldots \ldots$ & $-0,0.) 36^{* *}$ & $-0,0062 * *$ & $-0,0043^{* *}$ & $-0,0040^{* *}$ & $b_{3} \ldots \ldots \ldots$ & $-0,0050^{*}$ & $-0,0027 *$ & $-0,0053^{* *}$ & $-0,0040^{* *}$ \\
\hline$s \ldots \ldots$ & 0,368 & 0,287 & 0,312 & 0,332 & $s \ldots \ldots$ & 0,200 & 0,200 & 0,227 & 0,253 \\
\hline$R_{2} \ldots \ldots$ & 0,872 & 0,938 & 0,942 & 0,923 & $R_{2} \ldots \ldots$ & 0,959 & 0,976 & 0,960 & 0,955 \\
\hline
\end{tabular}

* Valeur significative au seuil de probabilité de $5 \%$. - ** Valeur significative au seuil de probabilité de $1 \%$. N.S. Valeur non significative au seuil 

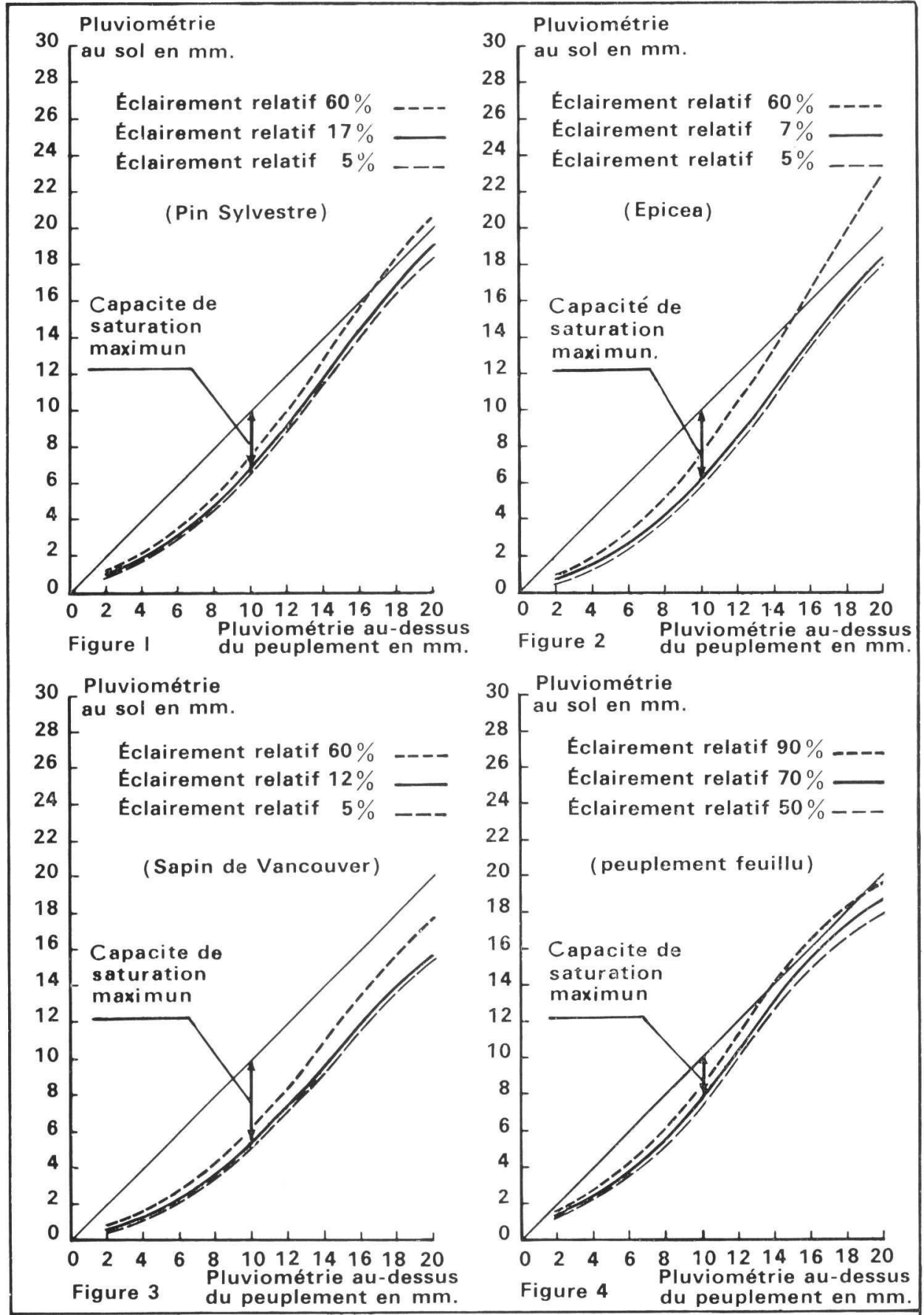

FIG. 1-4. - Précipitation sous couvert, en fonction des pluies incidentes. 


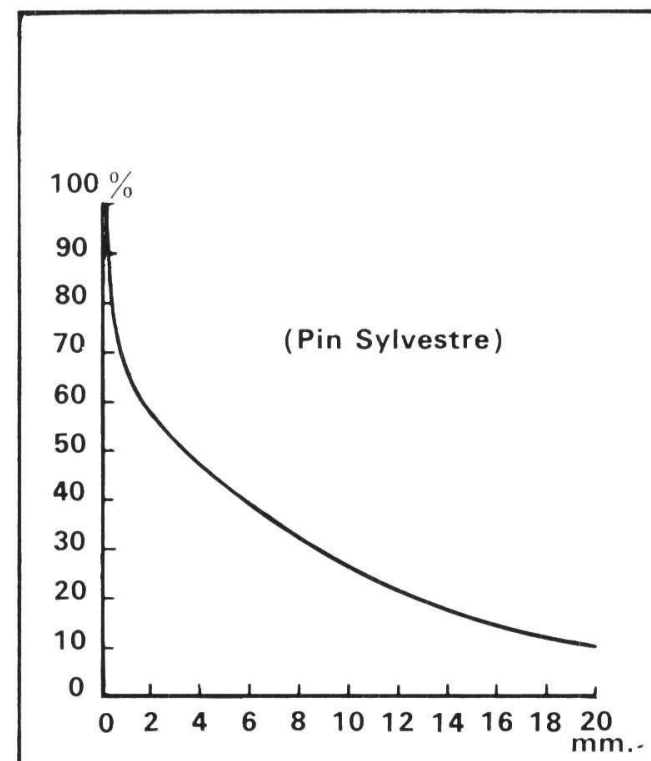

Figure 5

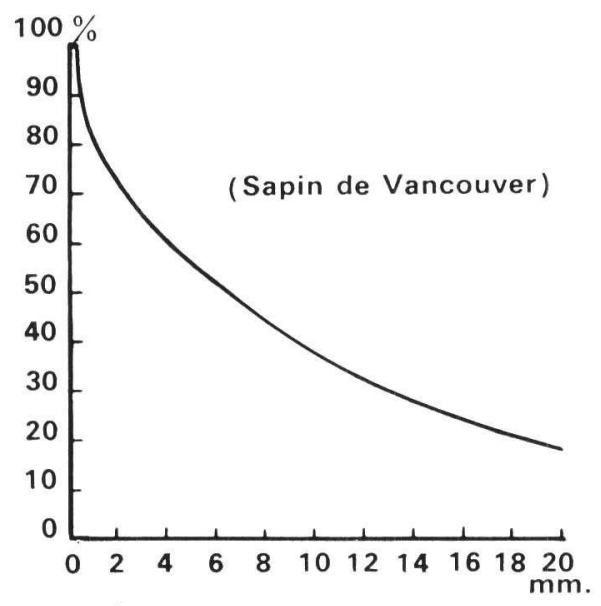

Figure 7

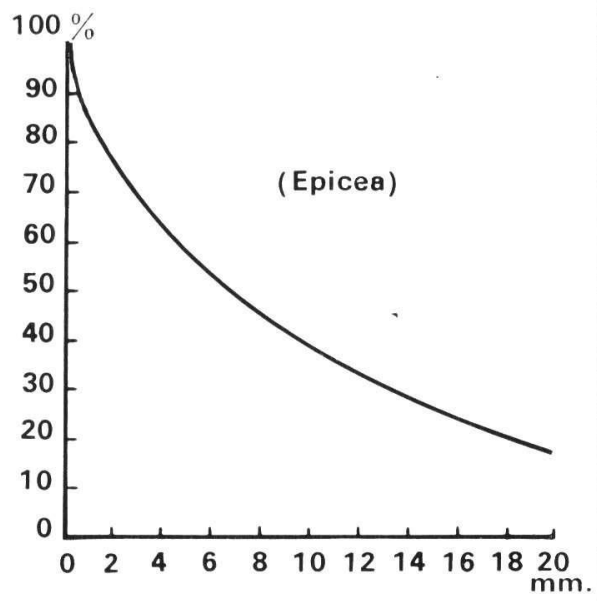

Figure 6

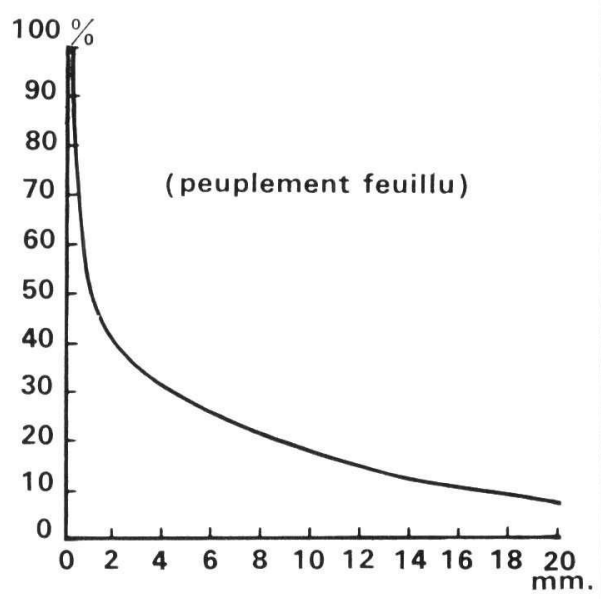

Figure 8

FIG. 5-8. - Interception en fonction de l'importance des précipitations. 
l'interception augmente jusqu'à une certaine valeur, puis se stabilise (Sapin de Vancouver) ou bien décroît lentement (pour les autres peuplements). Il y a là l'expression du phénomène de saturation du feuillage.

Le maximum atteint permet d'avoir une idée de la capacité maximum de saturation des couronnes des peuplements étudiés, soit : $4,5 \mathrm{~mm}$ chez Abies grandis, 4,0 $\mathrm{mm}$ chez Picea abies, 3,5 $\mathrm{mm}$ chez Pinus silvestris, 2,0 $\mathrm{mm}$ pour le peuplement feuillu. L'évaluation est du même ordre de grandeur que celle effectuée précédemment, ceci permet de recouper les résultats obtenus et de se rendre compte de la validité de la méthode utilisée pour évaluer la capacité de saturation des couronnes.

La diminution de l'interception avec l'augmentation des précipitations peut être attribuée à l'intervention du vent comme facteur perturbateur. Il semble que l'on puisse aussi faire intervenir le coefficient de captation des pluviomètres. Ce dernier est plus faible avec l'augmentation de la vitesse du vent. Ce qui se traduit par une diminution de l'évaluation de l'interception alors que, en réalité, cette diminution serait beaucoup moins importante.

L'interception est souvent exprimée en pourcentage des précipitations incidentes. Afin de faciliter les comparaisons, les ñgures 5 à 8 donnent l'allure des courbes pour les peuplements étudiés. L'interception exprimée en pourcentage diminue avec l'importance des précipitations.

L'analyse statistique a montré que la plupart des pluviomètres fixes dans les 4 peuplements étudiés donnaient une évaluation biaisée de la pluviométrie au sol.

Aux pluviomètres, présentant un biais, correspondaient des éclairements relatifs très éloignés de la moyenne. Au contraire, ceux qui ont fourni des valeurs non biaisées, présentaient un éclairement relatif proche de la moyenne du peuplement.

Finalement, 1'évaluation des précipitations arrivant directement au sol, avec des pluviomètres fixes, a été inférieure à l'évaluation réalisée avec les pluviomètres mobiles et les intervalles de confiance autour des moyennes sont plus importants (tableau 9).

A la suite de cette analyse, il apparaît que la disposition au hasard d'un nombre réduit de pluviomètres fixes ne donne pas une bonne évaluation de l'interception. En effet, la distribution de l'éclairement relatif n'est pas une distribution normale et dans la plupart des cas, les pluviomètres se localisent dans les zones à éclairement relatif faible donc à interception importante.

A la suite de ces résultats, nous sommes conduit à proposer une méthode d'évaluation de la pluviométrie au sol ou de l'interception :

- tout d'abord, faire un échantillonnage de l'éclairement relatif en utilisant le dispositif décrit plus haut ;

- ensuite, établir le polygone de distribution et répartir les pluviomètres dont on dispose, proportionnellement aux fréquences d'éclairement.

Dans chaque classe de fréquences, l'emplacement des pluviomètres est tiré au hasard. Dans le cas des feuillus, l'échantillonnage sera effectué l'été.

Les données de l'expérimentation ont permis de calculer le nombre de pluvio- 


\section{TABLEaU 9}

Evaluation des précipitations au sol à l'aide des pluviomètres mobiles et des pluviomètres fixes

\begin{tabular}{|c|c|c|c|c|c|c|c|}
\hline \multirow{2}{*}{$\begin{array}{l}\text { Espèces } \\
\text { Période }\end{array}$} & \multicolumn{2}{|c|}{ Pinus silvestris } & \multicolumn{2}{|c|}{ Picea abies } & \multicolumn{2}{|c|}{ Abies grandis } & Feuillus \\
\hline & $\begin{array}{c}\text { Mai } 1965 \text { à } \\
\text { avril } 1966\end{array}$ & $\begin{array}{c}\text { Mai } 1966 \text { à } \\
\text { avril } 1967\end{array}$ & $\begin{array}{l}\text { Mai } 1965 \text { à } \\
\text { avril } 1966\end{array}$ & $\begin{array}{l}\text { Mai } 1966 \text { à } \\
\text { avril } 1967\end{array}$ & $\begin{array}{l}\text { Mai } 1965 \text { à } \\
\text { avril } 1966\end{array}$ & $\begin{array}{l}\text { Mai } 1966 \text { à } \\
\text { avril } 1967\end{array}$ & $\begin{array}{c}\text { Juillet } 1966 \text { à } \\
\text { juin } 1967\end{array}$ \\
\hline Pluviomètres mobiles (en $\mathrm{mm}$ ) & $592,6 \pm 6,9^{*}$ & $510,2 \pm 6,4$ & $561,4 \pm 19,4$ & $479,7 \pm 18,9$ & $492,1 \pm 17,0$ & $433,4 \pm 15,0$ & $550,4 \pm 6,2$ \\
\hline Pluviomètres fixes (en mm) .. & $547,3 \pm 11,9$ & $464,6 \pm 8,1$ & $497,3 \pm 23,1$ & $420,3 \pm 20,5$ & $468,7 \pm 25,3$ & $407,5 \pm 19,6$ & $529,8 \pm 12,4$ \\
\hline
\end{tabular}

* Intervallè de confiance à $P=0,95$. 
mètres nécessaires pour évaluer les précipitations au sol, avec une erreur de $\delta=2,5 \%$ pour $\alpha=0,01$ (tableau $n^{0} 10$ ). Ceci, dans l'hypothèse d'une distribution correcte des pluviomètres.

La formule d'application utilisée était la suivante :

$$
n>t^{2}(n-1) \alpha \frac{s^{2}}{\delta^{2}}
$$

\section{TABLEAU 10}

Nombre de pluviomètres nécessaires à une bonne évaluation de la pluviométrie sous le couvert forestier

\begin{tabular}{l|c|c|c|c}
\hline \hline & $\begin{array}{c}\text { Pins } \\
\text { sylvestres }\end{array}$ & Epicéa & $\begin{array}{c}\text { Sapins de } \\
\text { Vancouver }\end{array}$ & Feuillus \\
\hline Nombre de pluviomètres ................ & 11 & 25 & 20 & 11 \\
\hline
\end{tabular}

\section{4. - DISCUSSION ET CONCLUSION}

Les phénomènes d'interception sont plus ou moins importants suivant les essences et les types de peuplement. Mais, de toute façon, ils interviennent dans le bilan hydrologique si l'on considère que les pourcentages annuels sont de $30 \%$ dans le peuplement de Pinus silvestris, de $34 \%$ pour Picea abies et $42 \%$ chez Abies grandis. Dans le peuplement feuillu, le pourcentage annuel est moindre : $17 \%$. Ceci est dû à la morphologie de la ramification et à la chute des feuilles pendant l'hiver. Bien qu'il soit difficile de comparer les chiffres obtenus avec ceux cités dans la littérature, les valeurs sont du même ordre de grandeur que celles citées par RUTTER (1965) et OviNGTON (1954). En raison des difficultés dévaluation de la pluviométrie incidente (coefficient de captation des pluviomètres) il est fort probable que l'interception soit sousestimée d'une façon générale.

L'écoulement, le long des troncs, est faible pour les résineux, mais une fraction importante des précipitations revient au sol de cette façon dans le peuplement feuillu. C'est d'ailleurs un phénomène assez général, ce qui a pour conséquence d'amener davantage d'eau aux sphères radiculaires des feuillus qu'à celles des résineux (NoRFALISE, 1959). D'ailleurs, dans certains types de végétations buissonnantes (chaparral, maquis), une grande partie des précipitations peut revenir au sol par les troncs (Hamilton et Rowe, 1949).

La hauteur d'eau nécessaire pour saturer le feuillage est estimée à $3,0 \mathrm{~mm}$ pour le peuplement de Pins sylvestres, 3,1 mm pour les Epicéas, 3,8 $\mathrm{mm}$ pour le peuplement de Sapins de Vancouver et 1,9 mm pour le peuplement feuillu. RutTer (1963) trouve des valeurs plus faibles $(2-3 \mathrm{~mm})$ pour un peuplement de Pins sylvestres de 16 ans et de 5,7 m de haut. Il est clair que ce paramètre dépend de l'importance des couronnes et de la structure du peuplement. Pour évaluer cette capacité de saturation, il est possible de pulvériser de l'eau sur des échantillons de rameaux feuillés et de branches, et en- 
suite d'effectuer une extrapolation pour un arbre. Mais, alors, on s'expose à des erreurs qui peuvent être considérables, car tous les rameaux n'ont pas la même morphologie, ni la même position dans l'espace.

Certains auteurs ont constaté que des quantités non négligeables d'eau pouvaient être ramenées au sol par égouttement des brouillards captés par les couronnes des arbres, le supplément pouvant ainsi aller jusqu'à $58 \%$ des précipitations annuelles (Kittredge, 1962). Ces condensations peuvent être décelables dans les régions côtières ou en montagne. A Nancy aucun égouttement de brouillard n'a pu être constaté, bien que des observations étaient effectuées spécialement. Cependant, il est permis de penser que par jours de brouillard une condensation légère s'est produite au niveau des feuilles et des branches, et cela a pu diminuer d'autant l'interception dans le cas où une pluie suivait le brouillard.

L'interprétation des données par la méthode de régression progressive a permis d'établir, pour chaque peuplement, l'équation liant les précipitations au sol à l'éclairement relatif et à la pluie incidente. Il s'agit d'une relation caractéristique de chaque peuplement.

A la suite de ces résultats, une méthode rationnelle d'évaluation de la pluviométrie au sol peut être envisagée : tout d'abord un échantillonnage de l'éclairement relatif, et ensuite une distribution des pluviomètres calquée sur la distribution de l'éclairement relatif. Il s'agit, dans ce cas, de pluviomètres à poste fixe. Cette méthode devrait être intéressante : d'une part, parce que des pluviomètres, en petit nombre, distribués au hasard à poste fixe, ne donnent pas une bonne évaluation de l'eau au sol, et d'autre part, parce qu'un dispositif de pluviomètres mobiles implique une organisation importante pour effectuer les mesures régulièrement.

Une question qui peut alors se poser, c'est la signification de l'éclairement relatif. Il est certain que pour une essence dans un peuplement donné, l'éclairement relatif est un paramètre synthétique. Il est permis de se demander si, pour une essence donnée et des éclairements relatifs identiques, mais correspondant à des peuplements différents, l'évaluation de la pluviométrie au sol sera la même. En d'autres termes, l'éclairement relatif est-il un paramètre suffisant pour exprimer la structure d'une essence forestière quand on s'adresse à des peuplements différents? Ce problème n'a pas été abordé, car il n'était pas possible de disposer de peuplements présentant les caractéristiques recherchées; il sera étudié dans un autre dispositif.

Les résultats précédents ont montré que l'on pouvait lier l'eau au sol à l'éclairement relatif et on a vu que les quantités arrivant au sol pouvaient être très différentes, suivant que l'éclairement relatif était fort ou faible, d'où, en fin de compte pour le sol, une alimentation variable en eau. Ici, apparaît le problème des régénérations naturelles en forêt. D'une façon générale, on lie l'apparition des régénérations à l'éclairement, mais la lumière n'est peut-être pas le seul facteur limitant, l'alimentation en eau pourrait aussi entrer en ligne de compte. Dans certaines stations présentant des sols à capacité de rétention faible (sol sableux par exemple) et un couvert à pouvoir d'interception important, il est possible qu'une mauvaise alimentation estivale en eau soit la cause des échecs de régénération. Le problème est d'autant plus 
complexe que l'essence à régénérer est une essence d'ombre (hêtre par exemple) et que, par conséquent, une ouverture du couvert n'améliore pas la situation.

A la suite des résultats obtenus, le problème le plus important qui se pose est la signification écologique de l'interception. S'agit-il d'une perte pure et simple, ou bien cette eau interceptée est-elle utilisée par les arbres? Quand on étudie l'interception, ce qui est évalué c'est la hauteur d'eau qui n'arrive pas au sol, c'est-àdire que ce n'est pas exactement ce qui est stocké au niveau des couronnes. VorgT (1964) a montré qu'une fraction importante de l'eau ( $40 \%$ ) qui s'écoulait le long des troncs était absorbée par l'écorce. STALFET (1944) a trouvé qu'une partie non négligeable de l'eau interceptée était absorbée par les feuilles de Picea abies. De son côté, RutTer (1965) n'observe pas d'absorption chez Pinus silvestris. La difficulté de mise en évidence de ces phénomènes et les différences dues aux espèces expliquent ces résultats.

Dans la mesure où une fraction de l'eau interceptée est localisée au niveau des feuilles, la transpiration est alors remplacée par une évaporation directe.

Au niveau de la feuille, l'E.T.P. est diminuée (BoucheT, 1963) et, en fin de compte, l'eau interceptée, bien que ne suivant pas le schéma classique sol-plante, est aussi efficiente, sinon plus, que si elle arrive au sol. Ces effets bénéfiques doivent alors être maxima dans les climats caractérisés par des pluies brèves, mais fréquentes, entrecoupées de période de beau temps; non seulement l'eau interceptée ne serait pas (( perdue ), mais alors, elle contribuerait à améliorer le rendement photosynthétique des arbres, car les stomates sont restés plus longtemps ouverts et la période d'assimilation a été accrue. Il est certain qu'une partie importante de l'eau interceptée est évaporée. Il s'agit là incontestablement d'un aspect très important du rôle modificateur du climat des formations forestières. En effet, l'évaporation de l'eau interceptée correspond à une consommation d'énergie, ce qui se traduit finalement par un abaissement de température de l'air (à titre d'exemple, remarquons que l'évaporation des $303 \mathrm{~mm}$, interceptés par les Sapins de Vancouver, correspond à $20 \%$ de l'énergie solaire). Il est alors permis de voir dans l'interception un élément décisif de l'action de la forêt sur le climat.

\section{Reçu pour publication en août 1968}

\section{REMERCIEMENTS}

Que soient remerciés ici :

- les membres de la Station de Biométrie du C.N.R.F. et notamment C. Millier.

- tous ceux qui ont collaboré avec nous : Techniciens, Dessinateur et Secrétaire.

\section{SUMMARY}

RAINFALL INTERCEPTION BY THE CANOPY

Our measures have been done to evaluate the importance of the interception in different forest stands which were approximately 30 years old.

The net annual interception has been found to be $30 \%$ for a Pinus silvestris stand, $34 \%$ for 
a Picea abies stand, $42 \%$ for an Abies grandis stand, and $17 \%$ for a deciduous stand concisting mainly of Fagus silvatica and Carpinus betulus.

The maximum amount of water necessary to saturate the crowns was respectively $3,0 \mathrm{~mm}$, $3,1 \mathrm{~mm}, 3,8 \mathrm{~mm}$ and $1,9 \mathrm{~mm}$ of rainfall in the case of Pinus silvestris, Picea abies, Abies grandis and the deciduous stand.

In each of these stands, we could establish an equation of the relationship between the precipitations under the canopy on one hand, and the incident rainfall above the canopy and the relative light intensity under the canopy on the other hand.

According to the results, we suggest a new and more accurate technique of evaluation of the through fall. The importance of the data have been discussed from an ecological stand point.

\section{ZUSAMMENFASSUNG}

\section{NiederschlagszurüCkHaLtung in WaldBestandeN}

In 30 jährigen Beständen verschiedener Holzarten wurden Niederschlag und Stammabfluss gemessen um die Bedeutung der verschiedenen Phänomene der Interzeption abzuschätzen.

In den einschichtigen und gleichaltrigen Beständen ergaben sich Jahresnettointerzeptionswerte in der Höhe von :

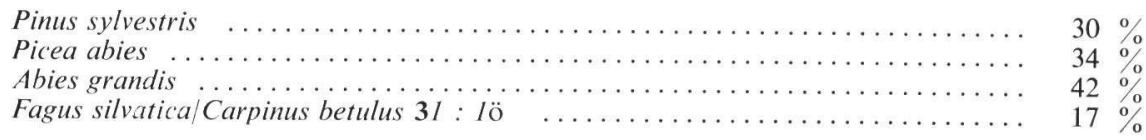

Die maximale Saturationskapazität konnte für die gleichen Bestände und in der selben Reihenfolge mit $3,0 \mathrm{~mm}, 3,1 \mathrm{~mm}, 3,8 \mathrm{~mm}$ und $1,9 \mathrm{~mm}$ bestimmt werden.

An Hand der mehr jährigen Messergebnisse konnte eine Stichprobenmethode erarbeitet werden, die, mit Hilfe einer Mehrfachregressionsgleichung, eine genauere objektive Bestimmung des Bestandesniederschlages als Funktion von relativer Beleuchtungsstärke und Freilandniederschlag erlaubt. Die ökologische Bedeutung der Niederschlagszurückhaltung wird eingehend besprochen.

\section{RÉFÉRENCES BIBLIOGRAPHIQUES}

Bouchet R.J., 1963. Evapotranspiration réelle, évapotranspiration potentielle et production agricole. Ann. agron., 14, 5, 743-824.

Carlisle A., Brown A.H.F. and White E.J., 1965, The interception of precipitation by oak (Quercus petrae) on a high rainfall site. Quart. J. For., 59, 2, 140-143.

Cepel N., 1967. Interzeption (= Niederschlagsverdunstung im Kroneuraum) in einen Buchen einem Eichen - und einem Kiefernbestand des Belgrader Waldes bei Instambul. Forstwissensch. Cbl., 5, 86, 301-314.

Cuif, 1913. Météorologie comparée agricole et forestière. Bull. Soc. Sci., 52, Nancy.

Eidmann F.E., 1959. Die Interception in Buchen und Fichtenbestanden, ergebnis mehyahriger untersuchungen im Rothaargebirge (Sauerland) Ass. internation. Hydrol. sci., 48, 5-26. Colloque Hannoversch. Münden.

Fautrat M., 1878. Observations météorologiques faites de 1877 à 1878, 44, Imprimerie nationale Paris.

Geiger R., 1965. The Climate near the ground. 611. Harvard University Press. Cambridge.

Guscina M.V., 1967. On the representativeness of precipitation gauge data. Meteorol. igidrol., 3, 101-106.

Hamilton E.L. and Rowe P.B., 1949. Rainfall interception by Chaparral in California Calif. For. Range exper. Stn. U.S. Forest. Serv., 3-35. 
HOOver M.D., 1953. Interception of rainfall in a young loblolly pine plantation. U.S. Forest. Serv. Southeastern Forest. exper. 21, 13.

Horton R.E., 1919. Rainfall interception. Month. Weather Rev. 47, 603-622.

Kittredge J., Loughead H.J. and MazuraK A., 1941. Interception and stemflow in a pine plantation. J. Forest., 39, 505-522.

Kittredge J., 1948. Forest influences. 394. McGraw Hill Book Co., New York.

LAW F., 1957. Measurements of rainfall interception and evaporation losses in a plantation of stika spruce trcess. Ass. internation. Hydrol. sci. Ass. géné. Toronto, 2, 397-411.

LeONARD R.E., 1966. Mathematical theory of interception. Internation. Symp. forest. Hydrol., 132136. Pergamon Press. Oxford.

Leyton L., Carlisle A., 1959. Measurement and interpretation of interception of precipitation by forest stands. Ass. internation. Hydrol. sci. 48, 111-119. Colloque Hannover Münden.

Leyton L., Reynolds E.R.C. and Thompson F.B. 1966. Rainfall interception in Forest and Moorland. Internation. Symp. forest. Hydrol., 163-177. Pergamon Press. Oxford.

Mathieu M., 1876. Relevé des observations de métćorologie comparée agricole et forestière faites aux environs de Nancy par l'Ecole Forestière. Atlas Météorologique. Observ. Paris., 8, 5.

Mitscherlich G., Moll. W., Kunstle E., Maurer P., 1966. Ertragskundlichökologische Untersuchungen im Rein-und Mischbestand. IV Niederschlag. Stammablauf und Bodenfenchtigheit. A.F.J.Z., 137, 1-13.

Molkanov A.A. 1960. The Hydrological role of forest. Izdatel' stvo Akademie Nauk SSSR. 407. Israël Programme for Scientific Translation. Jérusalem.

NaEgeli W., 1940. Licht messungen in Freiland und geschlossenen. Altholzbestanden Zurich.

Norrfalise A., 1959. Sur l'interception de la pluie par le couvert dans quelques forêts belges. Bull. Sci. r. Forest. Belg. 7.

Ovington F.D., 1954. A comparaison of rainfall in different woodlands. Forestry, 27, 41-53.

Poncelet L., 1959. Sur le comportement des pluviomètres. Publication $\mathrm{n}^{\circ} 10$ Série A. 58. Inst. $r$. Météorol. Belg.

Poncelet L., 1962. Sur le comportement des pluviomètres (comportement expérimental d'un écran de protection aérodynamique pour pluviomètre). Publication $\mathrm{n}^{\circ} 26$ Série A 14. Inst. r Météorol. Belg.

Reynolds E.R.C. and Leyton L., 1963. Measurements and significance of throughfall in forest stands. 127-141. In the Water Relations of Plants. Blackwell Scientific Publs. Oxford.

Reynolds E.R.C. and Henderson C.S., 1967. Rainfall interception by Beech, Larch and Norway Spruce. Forestry, 40, 2, 165-184.

Roussel L., 1963. Recherches théoriques et pratiques sur la répartition en quantité et en qualité sur la lumière dans le milieu forestier ; influence sur la végétation. Ann. Ec. nation. Eaux Forêts, 13, 2, 295-385.

RUTTER A.J., 1963. Studies in the water relation of Pinus silvestris in plantation conditions. I. Measurements of rainfall and interception. J. Ecol,, 51, 191-203.

SLATYER R.O., 1962. Measurements of precipitations interception by an arid zone plant community. (Acacia aneuraf. Muell). Recherches sur la zone aride. 181-192. Coll, de Montpellier.

SLAVIK B., 1962. Rain interception in deciduous forests. Recherches sur la zone aride. 193-199. Colloque de Montpellier.

SKAU G.M., 1964. Interception, througfall and stemflow in Utah and alligator juniper cover types of Northern Arizona. Forest Sci., 10, 283-287.

VoigT G.K., 1960. Distribution of rainfall under forest stands. Forest Sci., 6, 2-10.

Voigt G.K., ZwOLINSKi M.J., 1964. Absorption of stem-flow by bark of young Red and White pines. Forest Sci., 10, 277-282.

Wight C.L., 1941. An approach to the study of rainfall interception by forest canopies. J.S. Afri. Forest. Ass., 6, 54-70.

WILm H.G., 1943. Determining net rainfall under a conifer forest. J. agric. Res. 67, 12, 501-512.

ZINKE P.J., 1966. Forest. interception studies in the United States. Internation Symp. forest Hydrol. 137-160. Pergamon Press. Oxford. 